\title{
Titanium Disulfide Cathode Prepared Directly on the Titanium Plate ${ }^{\text {i }}$
}

\author{
Akira KiTANI*, Katsuaki KATOH*, Shoji YAMANAKA* \\ and Kazuo SASAKI*
}

\section{Introduction}

Recently, $\mathrm{Li} / \mathrm{TiS}_{2}$ cell is attracting attentions of many workers in the field of high energy density secondary batteries ${ }^{2}$. All the $\mathrm{TiS}_{2}$ cathodes reported in the literature have been prepared exclusively by hot-pressing a mixture of powdery $\mathrm{TiS}_{2}$ and some binders. However, the use of binders of organic polymers not only increases the internal resistance of a $\mathrm{TiS}_{2}$ cell but also leads to a decrease of energy density. In order to overcome these disadvantages, it is desirable to prepare the $\mathrm{TiS}_{2}$ cathode directly on the substrate of metallic titanium without using any binders.

In the previous paper, we prepared a thin layer of titanium sulfides by anodic oxidation of titanium plates in molten $\mathrm{Na}_{2} \mathrm{~S} \cdot 9 \mathrm{H}_{2} \mathrm{O}^{37}$. The main product obtained there was, however, titanium monosulfide and the expected product, $\mathrm{TiS}_{2}$, was not obtained. As a result, the output voltage of the lithium cell prepared by this method was one-half of the ordinary $\mathrm{Li}_{\mathrm{TiS}}$ cells.

In the present study, an attempt was made to prepare $\mathrm{TiS}_{2}$ directly on the surface of titanium plate by means of the thermal reaction. If this is possible, $\mathrm{TiS}_{2}$ cathodes can be prepared in one-step operation omitting the hot-press process and the resistance of the resultant electrode should be smaller.

\section{Experimental}

For the purpose of comparison, $\mathrm{TiS}_{2}$ was prepared in two different ways. One was prepared by reacting titanium plates $(5 \times 5 \times 0.5$ $\mathrm{mm}$ ) directly with sulfur at $700^{\circ} \mathrm{C}$ in a sealed quartz tube. In order to form the surface film only on one side of the titanium specimen,

* Department of Applied Chemistry, Hiroshima University (Saijo, Higashi-Hiroshima 724)

Key Words : Titanium Disulfide Cathode, Lithium Cell
Table 1 Preparation of $\mathrm{TiS}_{2}$ electrodes

\begin{tabular}{c|c|c|c}
\hline \multicolumn{2}{c|}{ Sample No. } & $\begin{array}{c}\text { Molar ratio } \\
(\mathrm{Ti}: \mathrm{S})\end{array}$ & $\begin{array}{c}\text { Heat treatment } \\
\text { at } 700^{\circ} \mathrm{C}(\mathrm{h})\end{array}$ \\
\hline \multirow{3}{*}{$\mathrm{MSE}$} & $1^{a>}$ & $1: 1$ & 18 \\
& $2^{b)}$ & $5: 1$ & 12 \\
& $3^{b)}$ & $10: 1$ & 6 \\
\hline \multirow{3}{*}{$\mathrm{PE}$} & $4^{c)}$ & $1: 2$ & 18 \\
& $5^{c)}$ & $1: 2$ & 12 \\
\hline
\end{tabular}

a) Product film peeled off the substrate body spontaneously. The film was directly mounted on one end of a brass rod to make the electrode, b) Base plate of $\mathrm{Ti}$ served as the collector electrode, c) Powdery product was hot-pressed at $300^{\circ} \mathrm{C}$ with $10 \%$ Tefon powder.

the other side of specimen was covered by asbestos during the heat treatment. The reaction time and the amount of sulfur to be reacted were varied to control the thickness of the $\mathrm{TiS}_{2}$ film as listed in Table 1 . In order to avoid confusion, we shall call this as the metal supported electrode (MSE). In this way, the lst sample was prepared by the reaction of titanium plate with equimolar amount of sulfur for 18 hours. A thick film (approximately 0.5 $\mathrm{mm}$ ) produced by this treatment was however readily peeled off from the substrate body without degradating the film structure. The film peeled off was used as it was for the cathode electrode (MSE 1) by mounting it directly on the brass rod lead. MSE's 2 and 3 were prepared by reducing both the molar ratio of sulfur to titanium and the reaction time. These two products were tightly attached to the substrate body and did not peel off. The thickness of $\mathrm{TiS}_{2}$ layer of MSE 3, which was the thinnest in the present study, was calculated to be 0.1 $\mathrm{mm}$ from the weight gain.

For all the three MSE's, X-ray diffractmetry revealed the reaction product to be $\mathrm{TiS}_{2}$ mainly. Besides this, a small amount of dimeric form 
$\left(\mathrm{Ti}_{2} \mathrm{~S}_{4}\right)$ was also detected. Scanning electron microscopy indicated that the crystal size of metal supported $\mathrm{TiS}_{2}$ was almost the same as powdery $\mathrm{TiS}_{2}$.

The other type of electrodes were made with the ordinary procedure to produce powdery $\mathrm{TiS}_{2}$. A given amount of spongy titanium was mixed with the stoichiometric amount of sulfur and heated in a quartz tube at $700^{\circ} \mathrm{C}$ for three different reaction times, which were made the same as in the preparation of MSE. Powdery $\mathrm{TiS}_{2}$ thus obtained was mixed with Teflon powder (10\% in weight) and pressed at $300^{\circ} \mathrm{C}$ with pressure of $2 \times 10^{6} \mathrm{~g} \cdot \mathrm{cm}^{-2}$ to form a pellet. We shall call this as the pellet electrodes (PE).

Either propylene carbonate (PC) or equivolume mixture of $\gamma$-butyrolactone (BL) and tetrahydrofuran (THF) were used as the solvent and $\mathrm{LiClO}_{4}\left(1 \mathrm{~mol} \cdot \mathrm{dm}^{-3}\right)$ as the supporting electrolyte. These solvents and electrolyte were purified and dried in a usual manner ${ }^{4}$. All experiments were done in a glove box under the argon atmosphere at room temperature.

\section{Results and Discussion}

Figure 1 shows the steady state polarization curves of two types of $\mathrm{TiS}_{2}$ electrode observed in two different solvent systems. Measure. ments were made galvanostatically. Results in

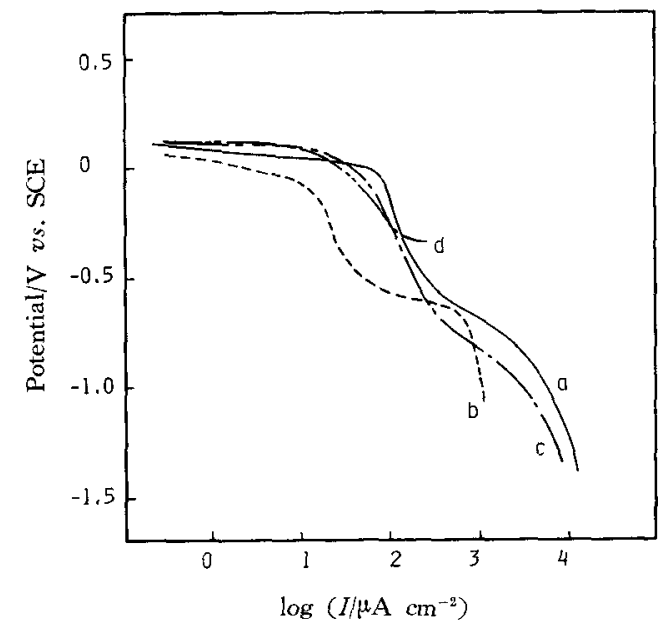

Fig. 1 Steady state polarization curves in PC (curve $b, d$ ) and BL-THF (curve $a, c$ )

Curves $a$ and $b$ were obtained with MSE 2, Curves $c$ and $d$ were obtained with PE 5 this figure exhibits typically a solvent effect on the cell performance with $\mathrm{TiS}_{2}$ electrodes. When $\mathrm{PC}$ was used as the solvent, active material began to detach from the electrode surface at a certain current density (curves $b$ and d). This phenomenon is attributed to the expansion of the layer distance of $\mathrm{TiS}_{2}$ as a result of intercalation of $\mathrm{PC}$ itself ${ }^{5}$. The threshold current density for the degradation was $2 \times 10^{-4}$ and $10^{-3} \mathrm{~A} \cdot \mathrm{cm}^{-2}$ for the pellet and metal supported electrodes, respectively. It is worth noting that the metal supported electrode is more resistant for the layer expansion than the pellet electrode. The threshold current density of the pellet electrode depends on both the amount of binder and pressing conditions. The degradation of electrode was inherent to the PC solvent system. Different behaviors are observed when solvent was changed. Curves $a$ and $c$ show this. In the equivolume mixture of $\mathrm{BL}$ and $\mathrm{THF}$ both the two types of electrodes, metal supported and pellet, were stable and no breakdown was observed upto $10^{-2} \mathrm{~A} \cdot \mathrm{cm}^{-2}$.

Curves in Fig. 2 show the effect of the nature of $\mathrm{TiS}_{2}$ electrodes prepared under several different conditions on the actual cell performance. In this measurement, cells were constructed

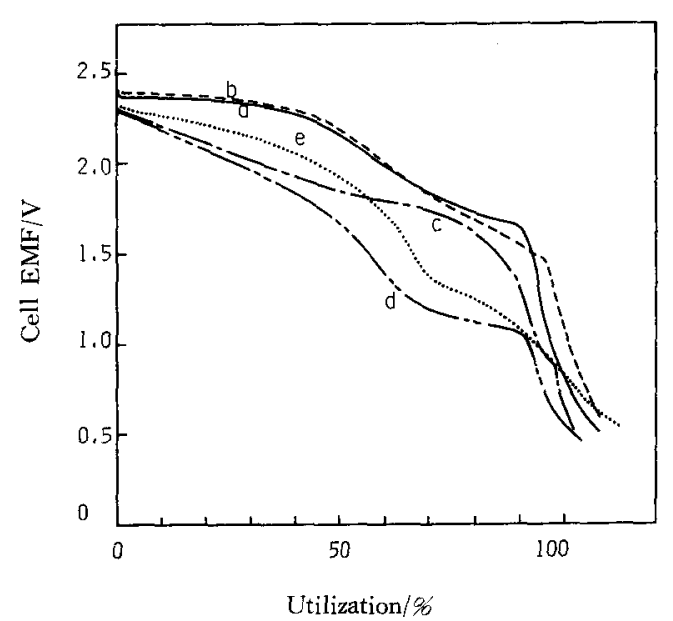

Fig. 2 Effect of nature of $\mathrm{TiS}_{2}$ cathodes on the cell performance

Discharge was made at constant load $(10 \mathrm{~K} \Omega)$, Solu-

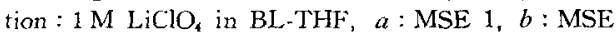
$3, c: \mathrm{PE} 4, d: \mathrm{PE} 6$, Curve $e$ was obtained with a pellet electrode made of the same material as PE 6 but contained no Teflon binder 
with $\mathrm{Li}$-anode and several types of $\mathrm{TiS}_{2}$ cathode. Solution was $1 \mathrm{M} \mathrm{LiClO}_{4}$ in BL-THF solvent. The change in output voltage was recorded with time during the discharge through a constant load $(10 \mathrm{~K} \Omega)$ and plotted against the fractional utilization of the cathodic active material, $\mathrm{TiS}_{2}$. In this figure, curves $a$ and $b$ were recorded with two different MSE's (MSE's 1 and 3), while $c, d$ and $e$ were with three kinds of pellet electrode. It is obvious from the figure that better performance is obtained with cells of MSE. The difference in voltage characteristics between MSE and PE may be attributed to the higher internal resistance of $\mathrm{PE}$ which contains organic binder. This is to some extent confirmed by comparing curves $d$ and $e$, which were obtained with two PE's made of the same $\mathrm{TiS}_{2}$ material (PE 6). Only difference was the use (curve $d$ ) and non-use (curve $e$ ) of binder. It is seen that the nonuse of binder certainly improves the output voltage to some extent but the improvement is still insufficient. It should be noted that the curve $a$ was obtained with MSE 1 , which was made of the film peeled off from the Ti-substrate and did not contain any binder either.

These results indicate that the use of binder in the preparation of $\mathrm{TiS}_{2}$ electrodes is quite undesirable. However, the disadvantage of using $\mathrm{PE}$ is not only related with this problem but also related with some other factors. As are represented by curves $b$ and $e$, the performance of a PE cell is still inferior even with an electrode free from binder. The reason of this may partly be attributed to that the intergranular contact resistance is still not negligible in PE. Another reason is probably attributed to the porous structure of PE. Because of the granular structure of a pellet the voidage is thought to be greater in PE than in MSE.

Usually, $\mathrm{TiS}_{2}$ powder is synthesized by reacting metallic titanium and sulfur for several days at $700^{\circ} \mathrm{C}$. On the other hand, the pellet electrodes examined here were all prepared with $\mathrm{TiS}_{2}$ which was heat treated only for 18 hours at the longest. This might be a reason of inferiority of the pellet electrodes. In order to make sure this point, we studied the effect of the duration of heat treatment. No improvement of the discharging characteristics was, however, observed upon increasing the reaction time from 18 to 48 or even to 168 hours. This shows that, as far as the practical use is concerned, the heat treatment can be reduced considerably. A 18 hours treatment, which is almost one-tenth of the time of usual heat treatment, seems to be sufficient.

In conclusion, metal supported electrodes have superior characteristics than the pellet electrodes made of powdery $\mathrm{TiS}_{2}$. Advantages can be summarized as follows.

1. Metal supported electrode can be prepared in one-step operation omitting the hot-press process.

2. Output voltage of a cell with this electrode is higher than that with the pellet electrode. This is probably due to the higher density of MSE than PE, which is made from powder by hot-press.

3. Since the use of any binder is unnecessary, the internal cell resistance becomes smaller.

4. It is also superior in the sense of the mechanical strength against the expansion of layer distance.

\section{Acknowledgement :}

This work was financially supported by the $\mathrm{Mi}$ nistry of Education (Grant No. 57850257).

\section{References :}

1) Studies on Electrochemical Cells Utilizing Intercalations part (II).

2) M.S. Whittingham, Prog. Solid. St. Chem. 12, 41 (1978).

3) A. Kitani, K. Kato, H. Nishihara, S. Yamanaka, K. Sasaki, Denki Kagaku 51, 391 (1983).

4) C.K. Mann, "Nonaqueous Solvents for Electrochemical Use", in A.J. Bard ed., "Electroanalytical Chemistry", Vol. 3, M. Dekker, New York, 1968.

5) S. Kikkawa, M. Koizumi, T. Yamamoto, "Proceedings of 46 th annual meeting of chemical society of Japan" p. 369 (1982).

(Received Mar. 14, 1983; Accepted May 4, 1983) 